\title{
Repair of Y-T Humeral Condyle Fractures with Locking Compression Plate Fixation
}

\author{
Felicity Moffatt ${ }^{1}$ Elvin Kulendra ${ }^{1,2}$ Richard L. Meeson ${ }^{1}$ \\ ${ }^{1}$ Department of Clinical Sciences and Services, Queen Mother \\ Hospital for Animals, Royal Veterinary College, North Mymms, \\ United Kingdom \\ ${ }^{2}$ North Downs Specialist Referrals, Bletchingley, United Kingdom \\ Address for correspondence Richard L. Meeson, MA, VetMB, PhD, \\ MVetMed, DipECVS, PGCertVetEd, FHEA, MRCVS, Department of \\ Clinical Sciences and Services, Queen Mother Hospital for Animals, \\ Royal Veterinary College, Hawkshead Lane, Hatfield, AL9 7TA, \\ United Kingdom (e-mail: rmeeson@rvc.ac.uk).
} Vet Comp Orthop Traumatol 2019;32:401-407.

\begin{abstract}
Keywords

- locking compression plate

- humeral

- condylar fracture

- Y fracture

- fracture fixation

- dogs

Objectives The aim of this study was to describe the use of locking compression plates (LCP) in Y-T humeral condyle fractures and to evaluate their clinical outcome. Methods This study involved a retrospective review, including clinical, radiographical and canine brief pain inventory outcome evaluation.

Results Eighteen consecutive dogs met the inclusion criteria, and 15/18 were considered to have humeral intracondylar fissure. Twelve of 18 dogs had simple fractures, and the remaining six had comminuted fractures. Postoperative radiographs revealed accurate intracondylar reconstruction (articular step defect [ASD] $<1 \mathrm{~mm}$ ) in 17/18 of patients. Short-term outcome was considered fully functional in $9 / 13$ and acceptable in $3 / 13$ patients. Complications were diagnosed in $2 / 13$; infection in one with resolution after antibiotic treatment, and one case of implant failure. Nine of 18 owners provided postoperative questionnaire responses (median 25; range: $14-52$ months) and $8 / 9$ clients perceived the treatment to have resulted in an excellent overall outcome.

Clinical significance Repair of Y-T humeral fractures with LCP allowed for hybrid fixation and monocortical screw placement in distal fracture fragments. There was no significant ASD at the intracondylar fracture line in most cases. ASD using combined medial and lateral approaches depends upon the accuracy of supracondylar reduction, particularly on the side that is reduced and stabilized first, and the use of locking screws may have been influential in minimizing primary loss of reduction, potentially maintaining the initial fragment reduction.
\end{abstract}

\section{Introduction}

Distal humeral condylar fractures, often described as Y-T fractures, are common in dogs and involve an intra-articular fracture of the humeral condyle with concurrent separation from the diaphysis. ${ }^{1-4}$ Rigid fracture fragment fixation and precise reconstruction of the articular surface are paramount to optimize functional outcome and limit development of osteoarthritis. ${ }^{1,5}$ Typically, the fragments are reduced via

\footnotetext{
(D) Richard L. Meeson's ORCID is https://orcid.org/0000-0002-89727067.
}

olecranon osteotomy or combined medial and lateral approaches, followed by rigid internal fixation. ${ }^{1,2}$ To date, their functional outcome has been assessed subjectively and results have been variable., ${ }^{1,3,6}$

There has been considerable interest in locking plate technology for fracture repair, with results demonstrating advantages under certain circumstances. $^{7-9}$ Cortical plating produces compression between the implant and the bone, relying on the generation of friction between plate and bone and between screw head and plate, ${ }^{10,11}$ whereas in locking plates, the screw is mechanically coupled to the plate. ${ }^{10}$ This

(c) 2019 Georg Thieme Verlag KG Stuttgart · New York
DOI https://doi.org/ $10.1055 / \mathrm{s}-0039-1691825$. ISSN 0932-0814.

April 10,2019 
minimizes the compressive forces exerted by the plate, thereby protecting periosteal vasculature and avoiding loss of reduction from imperfect plate contouring. ${ }^{10}$ The string of pearls ${ }^{R}$ locking implant has been previously used to stabilize Y-T fractures in 13 dogs, and this repair yielded good results, although additional surgery was required in $4 / 13 .{ }^{2}$ The locking compression plate (LCP) has the advantage of allowing either cortical or locking screw placement at each hole, ${ }^{7,10}$ facilitating the use of this implant as a compression plate, a locked internal fixator or a hybrid style fixation. ${ }^{10}$ The aim of this study was to report the outcomes of Y-T humeral condyle fractures in dogs repaired using LCP with a transcondylar screw.

\section{Materials and Methods}

Medical records of dogs presented to the Royal Veterinary College during the period 1st January 2010 to 1 st September 2016 with a distal Y-T humeral condylar fracture that was stabilized with a transcondylar screw and at least one LCP were reviewed. The following information was gathered for each patient: signalment, body weight, pertinent medical history/findings including suspected presence of humeral intracondylar fissure ${ }^{12}$ from intraoperative subjective assessment (sclerotic, relatively avascular intra-articular fracture surface, which was hard to drill), preoperative radiographs, implants placed, time to radiographic union (defined by cortical bridging and lack of visible fracture line), complications encountered, postoperative lameness and range of motion ( - Supplementary Appendix Table A, available in online version only). Ethical approval was granted by the institutional ethics committee (URN: M20160089).

\section{Surgical Technique}

All dogs had combined medial and lateral surgical approaches and internal fixation ${ }^{1,13}$ Typically, the medial supracondylar fracture was reduced first using a Kirschner wire(s) or lag $\operatorname{screw}(\mathrm{s})$, aiming for anatomic reduction. A suitable LCP was positioned medially, at the most distal aspect of the medial epicondyle, aiming for at least three screws distal to the fracture and three screws proximal to it. Minimal contouring was needed and consideration of screw placement was made to ensure that screws requiring angulation were placed first with cortical screws. Locking screws were placed thereafter, either bi- or monocortically. The medial side was then packed with saline moistened cotton gauze sponges to allow for the lateral approach to the humerus. ${ }^{1}$ An 'inside-out technique' transcondylar screw was placed (lag or positional by surgeon preference) aiming for screw diameter of 30 to $50 \%$ of the narrowest portion of the condyle. In the majority, a second LCP was contoured and applied, aiming for at least two bicortical screws distal and three proximal to the fracture line. The plate was variably placed between caudolateral and caudal sides of the humeral condyle, with the caudal aspect of the condyle reducing the requirement for plate contouring by twisting. Cortical screws were placed prior to locking.

\section{Radiographic Assessment}

Fracture configuration was assessed from the preoperative radiographs. The implants and repair were assessed on postoperative radiographs (Horos version 2.2.0 for Macintosh). The accuracy of articular surface reduction, and the resulting articular surface defect (ASD), was measured from digitally scaled caudocranial radiographs and graded as 0 (<1 mm), 1 (1-2 mm) or 2 (>2 mm). Plate size and length, screw type (cortical or locking) and number in each fragment and any additional implants were recorded. Radiographs were assessed for fracture configuration, healing and implant stability by a board-certified veterinary radiologist. Two authors, FM and RM (a board-certified small animal surgeon), assessed all radiographic parameters.

\section{Short-Term Follow-Up}

Radiographic follow-up was scheduled at 6 to 8 weeks and thereafter as required. Clinical records were evaluated for the short-term follow-up assessment, including range-of-motion, visual gait scored out of $10,{ }^{14}$ and for any instability, swelling, crepitus or any signs of discomfort. All clinical assessments were made by one of four board-certified small animal surgeons, or experienced surgical residents under their supervision. Overall clinical outcome defined using standardized definitions. ${ }^{15}$ For the purpose of this study, full function described those dogs with very mild or no reduction of elbow flexion and a lameness score of 0 to 2/10. Dogs with moderate reduction in elbow flexion and a lameness score of 3 to 6/10 were deemed to have acceptable function, and those with severe reduction in elbow flexion coupled with a lameness score of 7 to 10/10 were defined as having unacceptable function. Postoperative infection associated with the surgery included those within 12 months of surgery. ${ }^{16,17}$ Complications were defined as per current recommendations. ${ }^{15}$ Long-term follow-up from 12 months onwards was based on the canine brief pain inventory and an additional owner questionnaire. ${ }^{15,18}$

\section{Results}

Eighteen fractures met the inclusion criteria, with a short-term follow-up from 2.5 weeks to 7 months. The ages of the dogs ranged from 6 months to 8 years (median: 3 years 6 months), and bodyweight ranged from 8.5 to $35 \mathrm{~kg}$ (mean: $19.6 \mathrm{~kg}$ ). Breeds are reported in - Supplementary Appendix Table A, available in online version only. Humeral intracondylar fissure pathology was identified in 15/18 fractures. ${ }^{19}$ Twelve of 18 dogs had 'simple' fractures, and six had comminuted fractures; four condylar, one supracondylar and condylar, and one had severe supracondylar comminution with a mid-diaphyseal fracture of the humerus that had propagated through previous screw holes bilaterally (failed repair referred for revision). All dogs had open combined medial and lateral approaches, although one required additional olecranon osteotomy due to intra-articular comminution. The supracondylar region was stabilized with bilateral LCP in 16/18 dogs, an LCP (medially) with veterinary cuttable plate (laterally) in one dog and a single LCP (medially) with supracondylar stabilization on the lateral side using a Kirschner wire in one. Dogs weighing $<10 \mathrm{~kg}$ had 2.4 LCP bilaterally; dogs weighing 10 to $20 \mathrm{~kg}$ dogs had 2.7 LCP medially in 9/11 cases, two had 2.4 LCP, and the lateral component was stabilized with a $2.4 \mathrm{LCP}(n=6)$ or $2.7 \mathrm{LCP}$ 
Table 1 Medial and lateral implants showing range and median values in brackets

\begin{tabular}{|c|c|c|c|c|c|c|c|}
\hline & \multicolumn{6}{|l|}{ Screws } & \multirow{3}{*}{$\begin{array}{l}\text { Plate } \\
\text { Size }\end{array}$} \\
\hline & \multicolumn{3}{|c|}{ Distal to fracture } & \multicolumn{3}{|c|}{ Proximal to fracture } & \\
\hline & $\begin{array}{l}\text { Locking } \\
\text { screws }\end{array}$ & $\begin{array}{l}\text { Monocortical } \\
\text { screws }\end{array}$ & Overall & $\begin{array}{l}\text { Locking } \\
\text { screws }\end{array}$ & $\begin{array}{l}\text { Monocortical } \\
\text { screws }\end{array}$ & Overall & \\
\hline $\begin{array}{l}\text { Medial } \\
\text { implant }\end{array}$ & $1-4(3)$ & $1-5(3)$ & $2-5(4)$ & $1-5(3)$ & $0-4(0)$ & $3-5(4)$ & $7-14(9)$ \\
\hline $\begin{array}{l}\text { Lateral } \\
\text { implant }\end{array}$ & $0-4(2)$ & $1-4(3)$ & $2-4(3)$ & $1-5(3)$ & $0-4(1)$ & $2-5(3)$ & $6-14(7.5)$ \\
\hline
\end{tabular}

( $n=4)$. Dogs weighing 20 to $30 \mathrm{~kg}$ had 2.7 LCP medially $(n=4)$, and $3 / 4$ had 2.7 LCP laterally, one had a 2.4 LCP. Dogs weighing $>30 \mathrm{~kg}$ had a $2.7 \mathrm{LCP}$ applied medially in all cases $(n=2)$, and a 2.7 LCP $(n=1)$ or a $3.5 \operatorname{LCP}(n=1)$ applied laterally (-Supplementary Appendix Table A, available in online version only).

\section{Medial Implants and Lateral Implants See - Table 1.}

\section{Additional Implants}

The diameter of the single transcondylar screw inserted in each case was $4.5 \mathrm{~mm}(n=14), 3.5 \mathrm{~mm}(n=3)$ or $2.7 \mathrm{~mm}$ $(n=1)$. Additional supracondylar implants were placed in 9/18 cases, including a lag screw (cases $3,4,7,8,9$ ) or Kirschner wire (cases $2,14,16$ ) or both (case 18). Kirschner wires and tension band were placed for the olecranon osteotomy (case 17) (for full details see - Supplementary Appendix Table A, available in online version only).

\section{Accuracy of Fracture Reduction and Fracture Healing}

Postoperative radiographs taken immediately after surgery demonstrated ASD of 2 in one dog, ASD 1 in 4 dogs, and ASD 0 in 13/18 dogs (-Fig. 1, - Table 2). Suboptimal implant position and reduction in fragments (malalignment of the humeral metaphysis/diaphysis) were documented in one patient (case 15). This dog was a revision of a referred previously failed $Y$ fracture repair, and had a non-reconstructable supracondylar fracture region. Thirteen cases had short-term radiographic follow-up (range: 2.5 to 13 weeks), of which osseous union was evident in $7 / 13$ dogs by 6 to 8 weeks post-surgery. In a further four cases, evidence of fracture healing was apparent with stable implants. Three of these cases $(4,7,11)$ had full function on clinical assessment and did not require further appointments. One of these four (case 18) developed a major complication and was euthanatized. In 2/13 dogs (case 8 and 17), no evidence of healing was seen at the first postoperative appointment; however, subsequent radiographic assessment demonstrated complete osseous union at 5 and 7 months respectively.

\section{Clinical Assessment}

Short-term outcome was considered fully functional in 9/13 patients. This included case 8 , which has a grade $7 / 10$ lameness on the repaired limb at 2.5 weeks postoperatively with septic
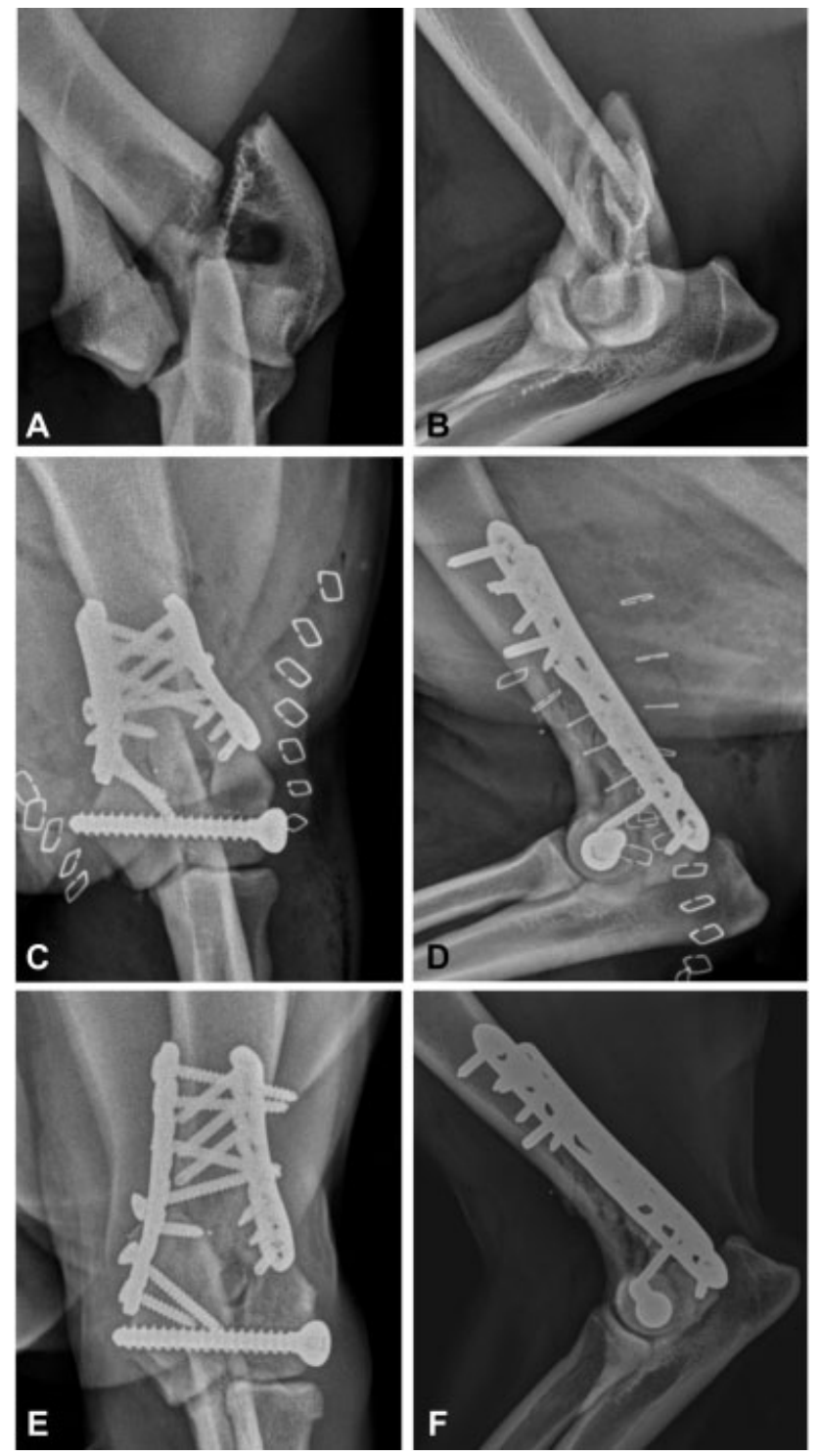

Fig. 1 Case 4 (Labrador Retriever) preoperative caudocranial (A) and mediolateral projections (B) showing simple condylar humeral fracture with a short lateral and long medial component. Immediate postoperative caudocranial (C) and mediolateral (D) views showing a medial 2.7-mm and lateral 2.4- $\mathrm{mm}$ locking compression plate, using hybrid fixation with a $4.5 \mathrm{~mm}$ transcondylar positional cortical screw. A small intra-articular gap persists consistent with humeral intracondylar fissure pathology and the articular step defect is $0.7 \mathrm{~mm}$. (E) Caudocranial and (F) mediolateral views at the 8-week postoperative stage showing ongoing intracondylar gap, with remodelling supracondylar fracture lines. 


\begin{tabular}{|c|c|c|c|c|c|c|c|c|c|c|c|c|c|c|c|c|c|c|}
\hline 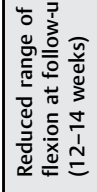 & 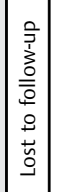 & 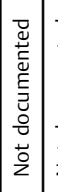 & 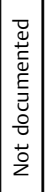 & 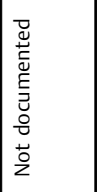 & 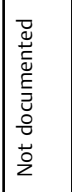 & 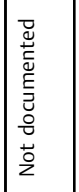 & 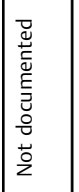 & \begin{tabular}{|l}
0 \\
$\overline{\tilde{L}}$ \\
$z$
\end{tabular} & 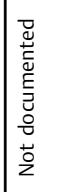 & 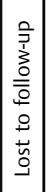 & 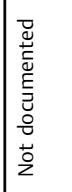 & 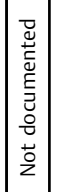 & 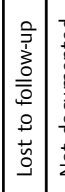 & 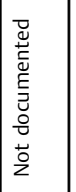 & 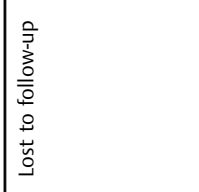 & 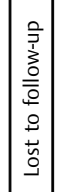 & 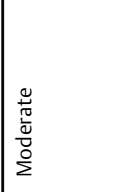 & 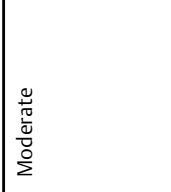 \\
\hline 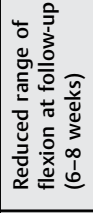 & 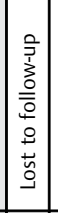 & 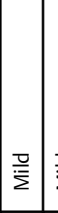 & $\frac{\bar{T}}{\bar{\Sigma}}$ & 总 & 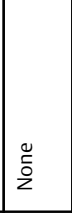 & $\frac{\tilde{D}}{\bar{\Sigma}}$ & $\frac{\bar{y}}{\bar{z}}$ & $\frac{\partial}{\bar{\Sigma}}$ & 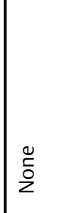 & 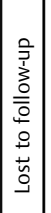 & $\frac{\overline{\underline{\nu}}}{\overline{\bar{\Sigma}}}$ & 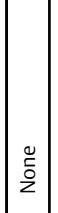 & 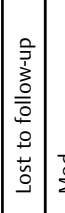 & $\frac{1}{2}$ & 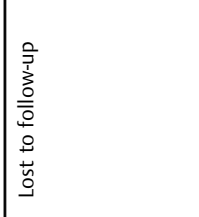 & 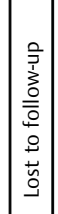 & 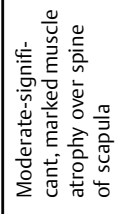 & $\frac{\bar{\nu}}{\overline{\bar{z}}}$ \\
\hline 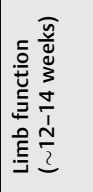 & 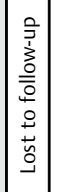 & 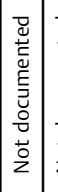 & 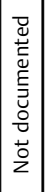 & 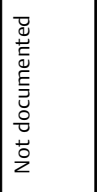 & 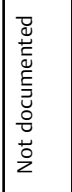 & 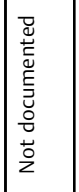 & 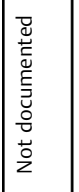 & 응 & 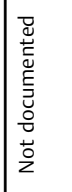 & 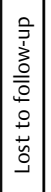 & 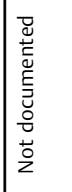 & $\frac{0}{0}$ & 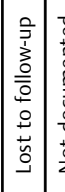 & 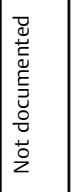 & 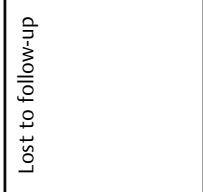 & 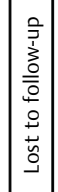 & 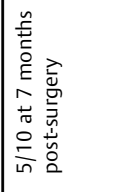 & $\frac{O}{m}$ \\
\hline 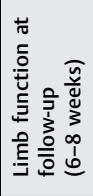 & 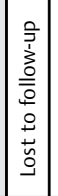 & $\frac{\circ}{0}$ & $\frac{0}{7}$ & $\frac{O}{N}$ & $\stackrel{ }{0}$ & $\frac{O}{N}$ & $\stackrel{ }{\circ}$ & 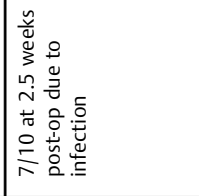 & $\frac{\circ}{\frac{2}{\gamma}}$ & 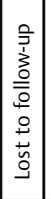 & $\frac{\stackrel{\rho}{m}}{m}$ & 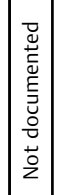 & 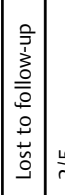 & $\frac{n}{N}$ & 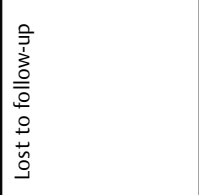 & 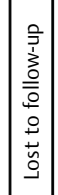 & 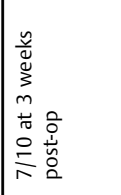 & $\frac{O}{N}$ \\
\hline 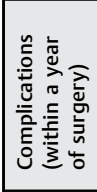 & 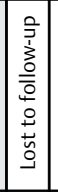 & $\mid$ & $\begin{array}{l}\frac{0}{0} \\
\frac{0}{2}\end{array}$ & 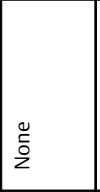 & 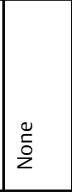 & 总 & 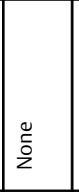 & 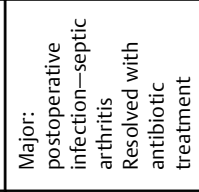 & 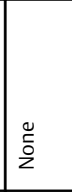 & 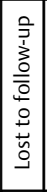 & 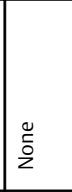 & $\mid \begin{array}{c}0 \\
\frac{\tilde{c}}{2} \\
z\end{array}$ & 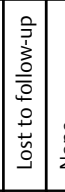 & 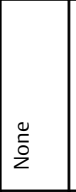 & 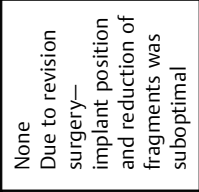 & 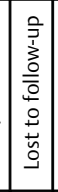 & 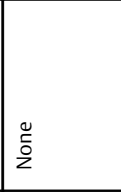 & 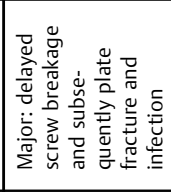 \\
\hline 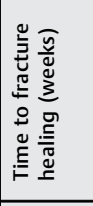 & 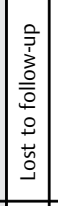 & \begin{tabular}{|l|l}
0 \\
0 \\
0
\end{tabular} & . & \begin{tabular}{|l}
+ \\
$\infty$
\end{tabular} & \begin{tabular}{|l}
+ \\
$\infty$ \\
\end{tabular} & \begin{tabular}{|l}
+ \\
$\infty$
\end{tabular} & $\infty$ & $\stackrel{+}{-\infty}$ & ${ }_{\infty}^{+}$ & 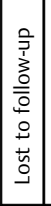 & 市 & $m$ & 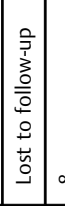 & $\infty$ & 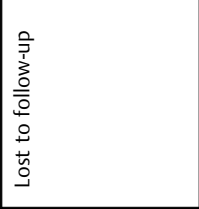 & 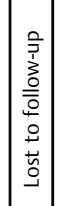 & 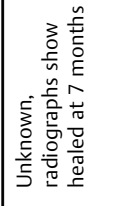 & 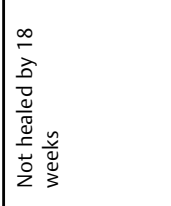 \\
\hline 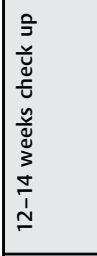 & 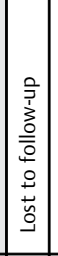 & 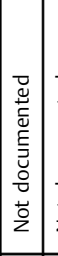 & 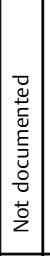 & 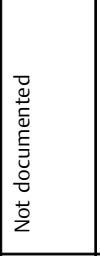 & 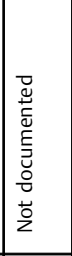 & 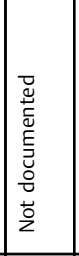 & 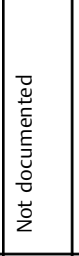 & 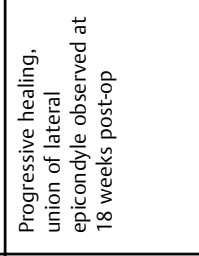 & 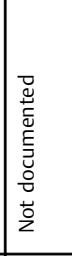 & 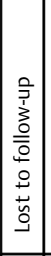 & 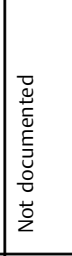 & 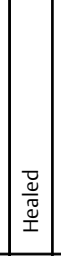 & 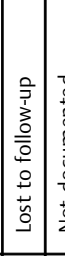 & 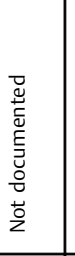 & 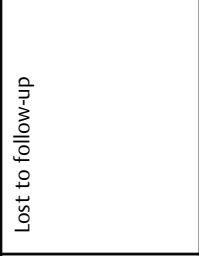 & 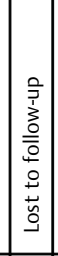 & 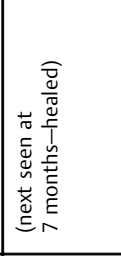 & 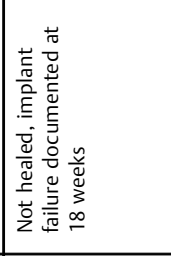 \\
\hline 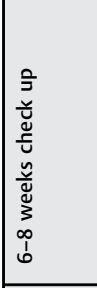 & 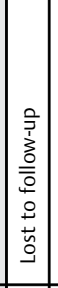 & 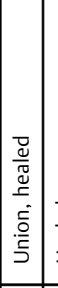 & $\begin{array}{l}\frac{\bar{v}}{\tilde{\mathbb{v}}} \\
\underline{\underline{I}}\end{array}$ & 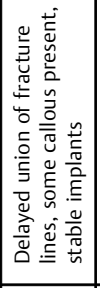 & 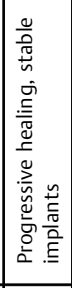 & 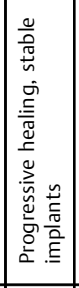 & 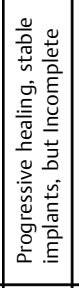 & 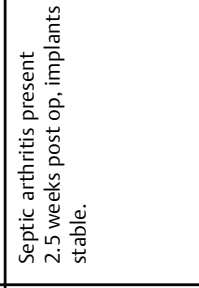 & 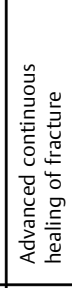 & 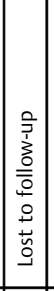 & 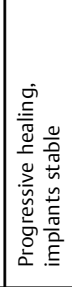 & 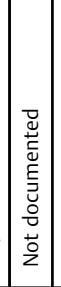 & 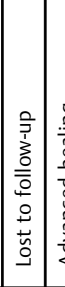 & 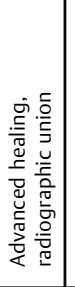 & 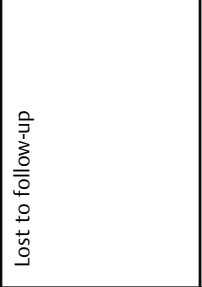 & 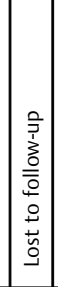 & 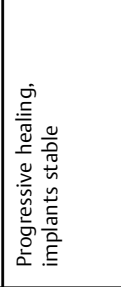 & 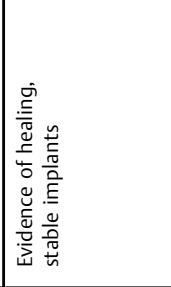 \\
\hline 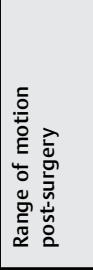 & 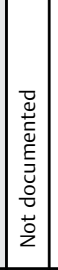 & 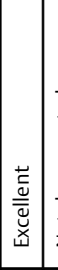 & 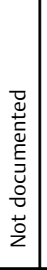 & 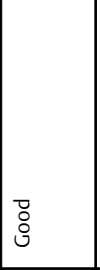 & 苂 & 总 & 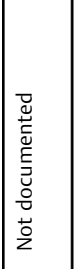 & 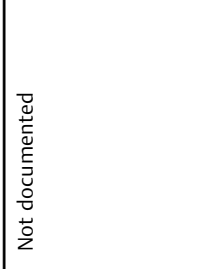 & $\begin{array}{l}\text { Do } \\
\text { Q }\end{array}$ & $\left|\begin{array}{|l}\vec{z} \\
\underline{0} \\
\underline{z}\end{array}\right|$ & 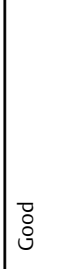 & $\mid \begin{array}{l} \\
\vdots \\
\vdots \\
\end{array}$ & 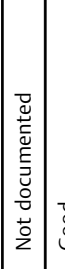 & $\begin{array}{l}\text { ¿. } \\
\text { ò }\end{array}$ & 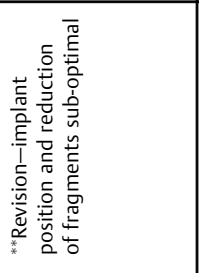 & 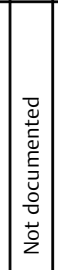 & 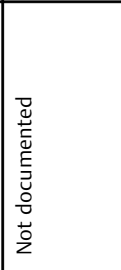 & 离 \\
\hline 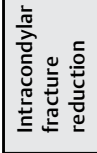 & $\mid \begin{array}{l}0 \\
\dot{z} \\
\dot{z}\end{array}$ & $\begin{array}{l}0 \\
0 \\
0 \\
\dot{z}\end{array}$ & $\mid \begin{array}{l}\overline{0} \\
\overline{\mathrm{z}}\end{array}$ & $\begin{array}{l}\circ \\
\text { 安 }\end{array}$ & 这 & 产 & 安 & 产 & 产 & $\mid \begin{array}{l}0 \\
0 \\
0 \\
\end{array}$ & 遂 & $\mid \begin{array}{l}0 \\
\dot{Q} \\
\dot{z}\end{array}$ & $\begin{array}{l}0 \\
\dot{0} \\
\dot{y}\end{array}$ & 安 & 安 & 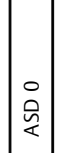 & 递 & 妾 \\
\hline 苞 & - & & & $\theta$ & in & 6 & r & $\infty$ & 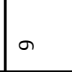 & 1 & $=$ & $\simeq$ & $\stackrel{m}{=}$ & 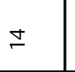 & $\stackrel{\llcorner}{\leftarrow}$ & $\underline{\square}$ & $=$ & 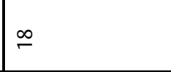 \\
\hline
\end{tabular}

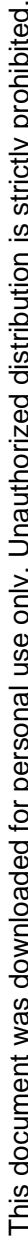


arthritis (with cytological confirmation) and made a full recovery (0/10 lame) after a 6 -week course of antibiotic medication. A further 3/13 had acceptable function. One dog had unacceptable function with significant reduction in elbow range of movement, marked muscle atrophy and was persistently grade 5/10 lame despite radiographic union at 7 months (case 17). This dog had intracondylar comminution and an additional olecranon osteotomy had been performed at surgery to facilitate surgical reduction.

\section{Complications}

Major complications were reported in 2/13 patients. Of the major complications, case 8 developed a postoperative infection 2.5 weeks post-surgery; however, no implant instability was noted and a full recovery was made following a 6 weeks course of antibiotic medication. The second dog (case 18) had a supracondylar comminuted $\mathrm{Y}$ fracture, and suffered delayed screw breakage and subsequently plate fracture and infection. Notably this dog had been treated with chronic steroid therapy for skin disease prior, and after fracture repair, exercise restriction was not enforced by the owner. This dog weighed $17.9 \mathrm{~kg}$, and was approximately $40 \%$ overweight based on breed average (-Fig. 2). Follow-up radiographs showed some transcondylar but little supracondylar remodelling. Short-term recovery was good, with a lameness score of $2 / 10$; only mild reduction in elbow flexion, stable implants and evidence of some intracondylar, but minimal supracondylar remodelling was observed at 7 weeks postoperative check. At 16 weeks, multiple fractured screws were noted, all in the distal medial fracture fragment. By 8 months, further screw and subsequent plate failure had occurred, and sampling revealed active infection. It was concurrently diagnosed with bilateral tarsocrural synovial osteochondromatosis and euthanatized.

\section{Long-Term Outcome}

Nine of 18 owners provided questionnaire responses at a median postoperative time of 25 months (range: 14-52) ( - Table 3). Owners rated the success of surgery as excellent in 8/9 dogs and good in 1/9. Impression of their dogs' overall quality of life was excellent in $7 / 9$, very good in $1 / 9$ and good in $1 / 9$. All owners were very satisfied with the treatment outcome, except for one who was 'satisfied'. Ongoing lameness or stiffness was reported in 3/9 dogs; two requiring long-term administration of non-steroidal anti-inflammatory drug medication and intermittent therapy with tramadol. Activity levels post-surgery were reported as very active in 4/9 dogs, active in $3 / 9$, average in $1 / 9$ and inactive in $1 / 9$. The canine brief pain inventory scores are reported in -Table 3.

\section{Discussion}

The outcome following repair of Y-T fractures using LCP was favourable; short-term outcome considered 'fully functional or acceptable' in 12/13 patients, and only 1/13 had unacceptable function. This is not dissimilar to other strategies of repair for Y-T fractures, ${ }^{1,2}$ although some studies have had a subjectively assessed outcome that was worse, with only 52 to $64 \%$ of
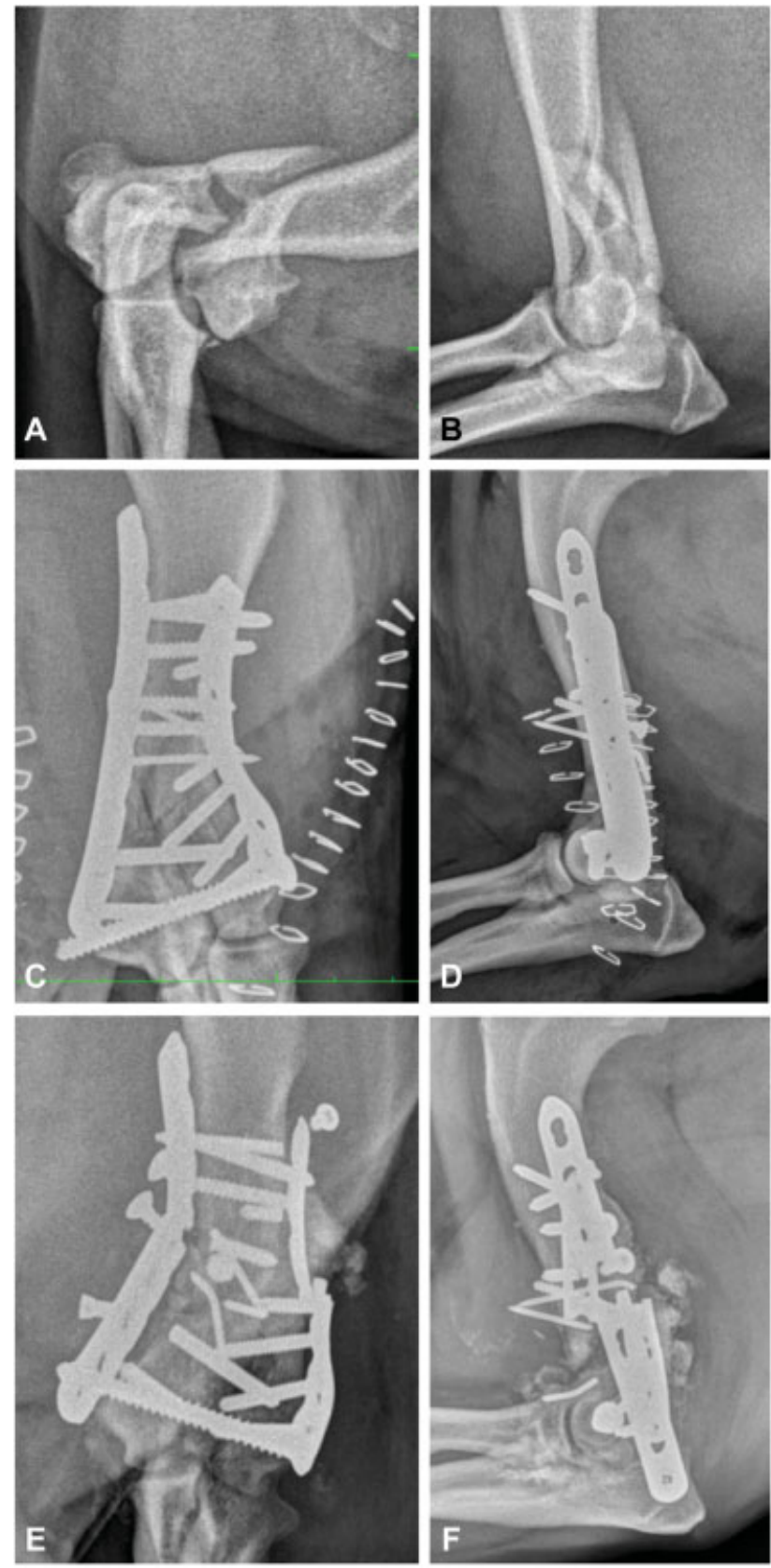

Fig. 2 Case 18 (French Bulldog), weighing $18 \mathrm{~kg}$ (breed standard 12.5 $\mathrm{kg}$ ), with a comminuted fracture, caudocranial $(A)$ and mediolateral views (B). Post-fracture repair with a medial $2.7 \mathrm{~mm}$ and lateral $2.4 \mathrm{~mm}$ locking compression plate, with additional lag screw and Kirschner wire stabilizing the supracondylar comminuted fragment, caudocranial (C) and mediolateral views (D). 8 Months later showing multiple screw failures, and bilateral plate fracture centred on the supracondylar region, caudocranial (E) and mediolateral views (F).

dogs achieving satisfactory results. ${ }^{3,6}$ When considering these types of clinical case series, it is important to acknowledge that subjective clinical assessment, which is known to be variable and susceptible to caregiver placebo, can make direct comparison difficult. ${ }^{20}$ However, this LCP study was aligned to current recommendations for outcome determination in clinical studies. $^{15}$

The bilateral approach ${ }^{1}$ was used in all cases and evaluation of postoperative radiographs revealed accurate intracondylar 
Table 3 Canine brief pain inventory mean postoperative pain severity scores and pain interference scores

\begin{tabular}{|c|c|c|c|c|c|c|c|c|}
\hline & $\begin{array}{l}\text { Success of } \\
\text { surgery }\end{array}$ & $\begin{array}{l}\text { Owner } \\
\text { impression } \\
\text { quality } \\
\text { of life }\end{array}$ & $\begin{array}{l}\text { Satisfied } \\
\text { with } \\
\text { treatment? }\end{array}$ & $\begin{array}{l}\text { Ongoing } \\
\text { lameness/ } \\
\text { stiffness }\end{array}$ & $\begin{array}{l}\text { Ongoing } \\
\text { medical } \\
\text { therapy }\end{array}$ & $\begin{array}{l}\text { Activity } \\
\text { levels post- } \\
\text { surgery }\end{array}$ & $\begin{array}{l}\text { Mean } \\
\text { post-op } \\
\text { pain } \\
\text { severity } \\
\text { scores }\end{array}$ & $\begin{array}{l}\text { Mean } \\
\text { interferences } \\
\text { scores }\end{array}$ \\
\hline Case 4 & Excellent & Very good & Very & $\begin{array}{l}\text { Yes, } \\
\text { permanently } \\
\text { lame, } \\
\text { osteoarthritis }\end{array}$ & $\begin{array}{l}\text { Yes: } \\
\text { Loxicom } \\
\text { Tramadol } \\
\text { Gabapentin }\end{array}$ & Inactive & 6.75 & 6.67 \\
\hline Case 6 & Excellent & Excellent & Very & $\begin{array}{l}\text { Yes, occasionally } \\
\text { (osteoarthritis), } \\
\text { but continues to } \\
\text { be very active }\end{array}$ & No & Very active & 0.0 & 0.33 \\
\hline Case 8 & Excellent & Excellent & Very & None & No & Active & 0.0 & 0.0 \\
\hline Case 9 & Excellent & Excellent & Very & None & No & Very active & 0.0 & 0.0 \\
\hline Case 10 & Excellent & Excellent & Very & None & No & Very active & 0.0 & 0.0 \\
\hline Case 13 & Excellent & Excellent & Very & None & No & Active & 0.0 & 0.0 \\
\hline Case 14 & Excellent & Excellent & Very & None & No & Active & 0.5 & 0.0 \\
\hline Case 15 & Good & Good & Satisfied & $\begin{array}{l}\text { Yes, at times } \\
\text { non-weight } \\
\text { bearing }\end{array}$ & $\begin{array}{l}\text { Yes: } \\
\text { Loxicom } \\
\text { Tramadol }\end{array}$ & Average & 5.0 & 7.5 \\
\hline Case 17 & Excellent & Excellent & Very & None & No & Very active & 0.0 & 0.0 \\
\hline
\end{tabular}

Note: Case $1,2,3,5,7,11,12,16,18$ not included due to lost to follow-up/owner not wanting to respond/dog deceased.

similar to the anatomic reduction from the string of pearls fixation with a bilateral approach. ${ }^{2}$ In contrast, $50 \%$ of dogs had poor reduction associated with this approach and cortical plating. ${ }^{1}$ Non-locking implants require highly accurate contouring to ensure sufficient friction between the plate and the underlying bone and to avoid primary reduction loss. ${ }^{11,21}$ Plating the distal humerus is particularly challenging due to the required twist and bend on the plate. If accurate plate conformation is not achieved, cortical plates could lead to a primary loss of reduction as the bone is pulled out of alignment toward the plate. ${ }^{2,21}$ In this LCP series, the majority of screws in the medial and lateral distal fracture fragments were placed as locking screws, potentially reducing disturbance of the reduction, and hence maintaining a good articular reduction ${ }^{22}$ from their fixed angle stability. ${ }^{7}$ This may have had particular benefit when first reducing the medial portion of the condyle, maintaining the supracondylar reduction, which if not correct will inhibit subsequent accurate intracondylar alignment when the lateral part is reduced. The LCP allowed for hybrid fixation that was employed in all cases in this series; however, it is important to ensure that the plate is accurately contoured and in contact with the bone in regions where non-locking screws are placed, and placing non-locking screws prior to locking screws. ${ }^{22}$ The string of pearls also had improved articular reconstruction, but differed from the LCP, as it used cortical screws, ${ }^{7}$ which were at a higher risk of breaking due to their smaller core diameter when compared with the locking screws. ${ }^{7}$ However, no such implant failures were reported by Ness. ${ }^{2}$

Notably, the majority of screws placed in the distal fragments were monocortical without any clear negative impact.
There remains debate as to the number of screws required proximal and distal to the fracture line in locking plate systems. It is thought that the increased stability of locking screws may allow for fewer cortices to be engaged in each bone segment while maintaining rigid fixation ${ }^{21}$ and recommendations vary from two to four cortices. ${ }^{22-24}$ Based on this study, the use of hybrid fixation including monocortical locking screws gave good clinical results.

Major implant-related complications were only diagnosed in a comminuted fracture in a small, overweight, chondrodystrophic breed dog that was suspected of having underlying humeral intracondylar fissure and was receiving chronic steroid therapy for skin disease. The comminution of the fracture coupled with the co-morbidities was probably significant factor for the delayed fracture healing, and implant breakage as postoperative reconstruction was deemed suitable. The other major complication was septic arthritis diagnosed at 2.5-week post-surgery and a 6-week course of antibiotic medications led to full recovery. Complete fracture union was achieved by 5 months post-surgery and the dog was reported to have excellent limb function with only mild reduction in elbow flexion.

Several of the cases were lost to follow-up; however, $13 / 18$ had equivalent follow-up as the 13 cases with string of pearls plates. ${ }^{2}$ This LCP study has the longest follow-up to date for Y-T fractures and further used a clinical metrology instrument. Other published work has had maximum 11 and 14 weeks, ${ }^{1,2}$ whereas all cases here had short-term median of 6 weeks follow-up and 50\% (9 cases) had long-term of 25 months (median), up to 52 months. Overwhelmingly, clients perceived the treatment to give an excellent overall 
outcome (88\%). Quality of life was perceived to be excellent in 7/9 cases and otherwise either very good or good. Ongoing lameness was seen in 3/9 of the dogs and was effectively managed using medical treatment and controlled exercise, allowing a good level of activity. This surgical technique gave a rapid return to activity post-procedure (4/9 dogs very active, 4/9 active and one dog inactive postoperatively) and achieved mostly excellent results long-term, with 8/9 of owners very satisfied with the outcome for their pet (one owner was 'satisfied').

\section{Conclusion}

In the present study, short-term outcome was excellent or adequate in most cases as was the long-term outcome. No dogs required additional surgery; however, the implant failure dog could have been a potential candidate for revision, although the pre-existing circumstances would remain a concern. The short-term outcome compared favourably with previous reports. ${ }^{1-3,6}$ Overall, the use of LCP, taking advantage of hybrid fixation and monocortical locking screws distally, gave good clinical outcomes and accurate articular alignment.

\section{Author Contribution}

Felicity Moffatt contributed to acquisition of data and data analysis and interpretation. Elvin Kulendra contributed to data analysis and interpretation. Richard L. Meeson contributed to conception of study, study design and data analysis and interpretation. All authors drafted, revised and approved the submitted manuscript.

\section{Funding}

No funding was provided for this manuscript

Conflict of Interest

The authors declare no conflict of interest.

\section{References}

1 McKee WM, Macias C, Innes JF. Bilateral fixation of Y-T humeral condyle fractures via medial and lateral approaches in 29 dogs. J Small Anim Pract 2005;46(05):217-226

2 Ness MG. Repair of Y-T humeral fractures in the dog using paired 'String of Pearls' locking plates. Vet Comp OrthopTraumatol 2009; 22(06):492-497

3 Anderson TJ, Carmichael S, Miller A. Intercondylar humeral fractures in the dog: a review of 20 cases. J Small Anim Pract 1990;31:437-442

4 Bardet JF, Hohn RB, Rudy RL. Fractures of the humerus in dogs and cats. A retrospective study of 130 cases. Vet Surg 1983; 12:73-77

5 Macias C, McKee WM. Articular and periarticular fractures in the dog and cat. In Pract 2003;25:446-465
6 Vannini R, Smeak DD, Olmstead ML. Evaluation of surgical repair of 135 distal humeral fractures in dogs and cats. J Am Anim Hosp Assoc 1998(b)24:537-545

7 DeTora M, Kraus K. Mechanical testing of $3.5 \mathrm{~mm}$ locking and non-locking bone plates. Vet Comp OrthopTraumatol 2008;21 (04):318-322

8 Frigg R. Locking compression plate (LCP). An osteosynthesis devise based on the dynamic compression plate and the point contact fixator (PC Fix). Injury 2001;32(Suppl 2):63-66

9 Schütz M, Südkamp NP. Revolution in plate osteosynthesis: new internal fixator systems. J Orthop Sci 2003;8(02):252-258

10 Wagner M. General principles for the clinical use of the LCP. Injury 2003;34(Suppl 2):B31-B42

11 Johnson KA. Locking plates - the ultimate implant? "Nothing holds up the progress of science so much as the right idea at the wrong time." Vincent de Vigneaud, Canada (1978). Vet Comp OrthopTraumatol 2009;22(02):I-II

12 Moores AP, Moores AL. The natural history of humeral intracondylar fissure: an observational study of 30 dogs. J Small Anim Pract 2017;58(06):337-341

13 Piermattei DL. The Forelimb. In: Piermattei DL, Johnson KA, eds. An Atlas of Surgical Approaches to the Bones and Joints of the Dog and Cat. 4th ed. Philadelphia, PA: Saunders; 2004:149-275

14 Sumner-Smith G. Gait analysis and orthopaedic examination. In: Slatter D, ed. Textbook of Small Animal Surgery. 2nd ed. Philadelphia, PA: WB Saunders; 1993:1577-1586

15 Cook JL, Evans R, Conzemius MG, et al. Proposed definitions and criteria for reporting time frame, outcome, and complications for clinical orthopedic studies in veterinary medicine. Vet Surg 2010; 39(08):905-908

16 Bennett D, Taylor DJ. Bacterial infective arthritis in the dog. J Small Anim Pract 1988;29:207-230

17 Brown DC. Wound infections and antimicrobial use. In: Tobias KM, Johnston SA, eds. Veterinary Surgery Small Animal Vol 1. Missouri: Elsevier Saunders; 2012:135-139

18 Brown DC, Boston RC, Coyne JC, Farrar JT. Development and psychometric testing of an instrument designed to measure chronic pain in dogs with osteoarthritis. Am J Vet Res 2007;68 (06):631-637

19 Moores A. Humeral condylar fractures and incomplete ossification of the humeral condyle in dogs. In Pract 2006;28:391-397

20 Conzemius MG, Evans RB. Caregiver placebo effect for dogs with lameness from osteoarthritis. J Am Vet Med Assoc 2012;241(10): 1314-1319

21 Filipowicz D, Lanz O, McLaughlin R, Elder S, were S. A biomechanical comparison of 3.5 locking compression plate fixation to 3.5 limited contact dynamic compression plate fixation in a canine cadaveric distal humeral metaphyseal gap model. Vet Comp OrthopTraumatol 2009;22(04):270-277

22 Egol KA, Kubiak EN, Fulkerson E, Kummer FJ, Koval KJ. Biomechanics of locked plates and screws. J Orthop Trauma 2004;18(08):488-493

23 Hertel R, Eijer H, Meisser A, Hauke C, Perren SM. Biomechanical and biological considerations relating to the clinical use of the Point Contact-Fixator-evaluation of the device handling test in the treatment of diaphyseal fractures of the radius and/or ulna. Injury 2001;32(02, Suppl 2):B10-B14

24 Gautier E, Sommer C. Guidelines for the clinical application of the LCP. Injury 2003;34(02, Suppl 2):B63-B76 